\title{
Perfil Psicomotor de Crianças com Transtorno do Espectro Autista em Maceió/AL
}

\author{
Psychomotor Profile of Children With Autism Spectrum Disorder in Maceió/AL
}

Perfil Psicomotor de los Niños con Trastorno del Espectro Autista en Maceió/AL

\author{
Clarissa Cotrim dos Anjos ${ }^{1}$ \\ Jessica Santos de Lima ${ }^{2}$ \\ Renata de Oliveira Araújo ${ }^{3}$ \\ Anne Kelly de Melo Calheiros ${ }^{4}$ \\ José Erickson Rodrigues ${ }^{5}$ \\ Sandra Adriana Zimpel ${ }^{6}$
}

\section{Resumo}

Objetivo: Traçar o perfil psicomotor das crianças com Transtorno do Espectro Autista. Métodos: Trata-se de uma pesquisa descritiva e transversal, realizado em dois centros especializados para o tratamento de crianças com Transtorno do Espectro Autista, na cidade de Maceió-AL, cuja amostra foi de 30 crianças com idade compreendida entre 2 e 11 anos, de ambos os gêneros, com diagnóstico de Transtorno do Espectro Autista leve, descrito no prontuário. Aplicou-se, inicialmente, um instrumento de coleta de dados com os responsáveis pela criança. Posteriormente foi realizada avaliação da criança em relação à escala de desenvolvimento motor de Rosa Neto. A análise dos dados foi realizada por meio da estatística descritiva. Resultados: A média da idade motora geral foi de $70 \pm 29,3$ em meses, sendo esta inferior à idade cronológica que foi de $88,5 \pm 27$ em meses, o que equivale a 7 anos e 3 meses. Verificou-se que as crianças com Transtorno do Espectro

${ }^{1}$ Fisioterapeuta Graduada pela UNCISAL, Especialista em Ciências da Saúde e Neurofuncional, Docente dos Curso de Fisioterapia do Centro Universitário Cesmac e na UNCISAL, Mestranda em Ensino da Saúde pela FAMED-UFAL. Autora correspondente: Rua São Francisco de Assis, 392/702, Edf Porto Fino, Jatiúca. CEP 57035-680. Maceió-AL, Brasil. E-mail: clacotrimanjos@gmail.com

${ }^{2,3}$ Fisioterapeuta, Graduada pelo Centro Universitário Cesmac.

${ }^{4,5}$ Graduanda em Fisioterapia pelo Centro Universitário Cesmac.

${ }^{6}$ Fisioterapeuta, Mestre em Ensino em Ciências da Saúde pela UNIFESP, Docente dos Cursos de Fisioterapia no Centro Universitário CESMAC e na UNCISAL.

Recebido: Dez/2016 - Aceito: Abr/2017. 
Autista avaliadas obtiveram menor idade motora para os elementos psicomotores de organização temporal e esquema corporal e maior para motricidade global e equilíbrio. $\mathrm{O}$ escore do quociente motor geral das crianças com Transtorno do Espectro Autista encontrado foi de $77,3 \pm 16,9$, sendo as mesmas classificadas em um nível inferior, na escala de desenvolvimento motor de Rosa Neto. Conclusão: Verificou-se que a Fisioterapia deve utilizar-se dos princípios da psicomotricidade como fundamento sensório-perceptivo-motor durante o tratamento das crianças com Transtorno do Espectro Autista, visto que as mesmas apresentam alterações nos aspectos psicomotores.

\section{Descritores: Transtorno Autístico; Desempenho Psicomotor; Desenvolvimento Infantil.}

\section{Abstract}

Objective: Trace the psychomotor profile of children with Autistic Spectrum Disorder. Methods: This is a descriptive and cross-sectional study carried out in two specialized centers for the treatment of children with Autistic Spectrum Disorder in the city of Maceió, whose sample consisted of
30 children aged between 2 and 11 years of age, of both genders, diagnosed with mild Autistic Spectrum Disorder described in the medical record. Initially, a data collection instrument was used with those responsible for the child. Subsequently, the child was evaluated in relation to the Rosa Neto motor development scale. Data analysis was performed using descriptive statistics. Results: The mean age of the general motor age was $70 \pm 29.3$ in months, which was lower than the chronological age, which was $88.5 \pm 27$ months, which is equivalent to 7 years and 3 months. It was verified that the children with Autistic Spectrum Disorder evaluated had lower motor age for the psychomotor elements of temporal organization and corporal scheme and greater for global motor and balance. The overall motor quotient score of the children with Autistic Spectrum Disorder was $77.3 \pm 16.9$, and they were classified in a lower level in the Rosa Neto motor development scale. Conclusion: It was verified that Physiotherapy should use the principles of psychomotricity as a sensory-perceptive-motor basis during the treatment of children with Autistic Spectrum Disorder, since they present changes in the psychomotor aspects. 


\section{Descriptors: Autistic disorder; Psychomotor performance; Child development.}

\section{Resumen}

Objetivo: Trazar el perfil psicomotor de los niño con Trastorno del Espectro Autista. Métodos: Se trata de un estudio descriptivo, trasversal, realizado en dos centros especializados para el tratamiento de los niños con Trastorno del Espectro Autista de la ciudad de Maceió-AL, se incluyen en el estudio, 30 niños con edad comprendida entre los 2 y 11 años, de ambos los géneros, y con el diagnostico de Trastorno del Espectro Autista de grado leve, descritos en los datos de la historia clínica. Se ha aplicado un instrumento para recoger los datos con los responsables de los niños. Enseguida fue realizada la avaluación de los niños en relación a la escala del desarrollo motor del Rosa Neto. El análisis de los datos fue realizado por medio de la estadística descriptiva. Resultados: La edad motora tiene una media de $70 \pm 29,3$ en meses, mientras sea inferior a la edad cronológica que fue de 88,5 \pm 27 meses de edad, lo que equivale a los 7 años y 3 meses de edad. Se ha verificado que los niños con
Trastorno del Espectro Autista analizados, obtuvieran menor edad motora para los elementos psicomotores de la organización temporal y de los esquemas corporales y mayores resultados para la motricidad global y del equilibrio. El escore de los coeficientes motores generales de los niños con Trastorno del Espectro Autista fue de 77,3 $\pm 16,9$, la misma clasificada en un nivel inferior en la escala de desarrollo motor de Rosa Neto. Conclusión: Se ha verificado que la fisioterapia debe utilizarse de los principios de la psicomotricidad como fundamento sensorio-perceptivo-motor en el tratamiento de los niños con Trastorno del Espectro Autista, pues los mismos presentan alteraciones en los aspectos psicomotores.

Descriptores: Trastorno Autístico; Desempeño Psicomotor; Desarrollo infantil.

\section{Introdução}

O Transtorno do Espectro do Autista é uma condição neurodesenvolvimental que se apresenta no início dos primeiros anos de vida da criança. Possui etiologia ainda desconhecida, mas pesquisas têm 
apontado correlatos neurobiológicos e genéticos $^{(1)}$. Dados epidemiológicos demonstram uma prevalência de um em cada 150 nascimentos, sendo que esta vem aumentando nas últimas décadas ${ }^{(1)}$. $\mathrm{Na}$ ausência de um marcador biológico, o diagnóstico de Transtorno do Espectro do Autismo permanece clínico. O Manual Diagnóstico e Estatístico de Transtornos Mentais utiliza as três seguintes dimensões diagnósticas: interação social, comunicação e comportamentos repetitivos e estereotipados, sendo esses os critérios que constituem a "tríade diagnóstica"(1).

As crianças com Transtorno do Espectro do Autismo podem apresentar uma diversidade de alterações do ponto de vista motor, dentre elas: equilíbrio, marcha, destreza manual, habilidades com bola e controle de objetos ${ }^{(2)}$. Assim, deve-se entender o desenvolvimento motor como um processo de mudanças complexas e interligadas, das quais participam todos os aspectos de crescimento e maturação dos sistemas orgânicos, encontrando-se na criança com Transtorno do Espectro do Autismo, alteradas essas interligações $^{(3)}$. Isso porque cada criança apresenta um padrão específico de desenvolvimento, que depende tanto das estruturas orgânicas quanto das influências ambientais, porém, na criança com Transtorno do Espectro do Autismo, parece que essa estruturação orgânica está inadequada ${ }^{(4)}$.

Dentro das prioridades do desenvolvimento infantil, encontra-se a Psicomotricidade, haja vista ser um elemento que se relaciona com a maturação neurológica. Quando não ocorre um amadurecimento neurológico ideal, a evolução psicomotora estará prejudicada, o que nas crianças com Transtorno do Espectro do Autismo poderá ser bastante evidente no tocante ao desenvolvimento dos elementos psicomotores visto que necessitam de uma boa exploração do meio externo bem como do conhecimento do meio interno, sendo esse déficit representado por sinais de isolamento, perda da iniciativa, ausência de apetência de aprendizagem e oposição, dificuldade de concentração na atividade e comunicação verbal $^{(5,6)}$.

As crianças com Transtorno do Espectro do Autismo geralmente apresentam um comprometimento dos elementos psicomotores devido ao fato da integração de todas as funções necessárias para um bom desenvolvimento da criança como: as funções cognitivas, sócio-emocionais, simbólicas, psicolinguísticas e motoras, promovendo, dessa maneira, a 
capacidade de a criança ser e agir dentro de um contexto psicossocial ${ }^{(7,8)}$.

A atuação do Fisioterapeuta no campo da Psicomotricidade ainda é pouco explorada, no entanto é essencial para o processo de amadurecimento e evolução das crianças com Transtorno do Espectro do Autismo. Com a utilização da Psicomotricidade como instrumento, poderão prevenir e minimizar os déficits dos componentes psicomotores que são esperados encontrar nessas crianças favorecendo assim o desenvolvimento das habilidades funcionais.

Portanto, conhecer o perfil psicomotor das crianças com Transtorno do Espectro do Autismo se faz necessário, visto que, prováveis déficits psicomotores podem ser encontrados devido a um não amadurecimento neurológico de forma adequada. Acredita-se que o perfil psicomotor das crianças com Transtorno do Espectro do Autismo seja inferior ao de uma criança com desenvolvimento típico. Assim, o objetivo deste estudo foi de traçar o perfil psicomotor das crianças com o Transtorno do Espectro do Autismo da cidade de Maceió/AL.

\section{Método} de um estudo descritivo transversal, realizado com crianças com Transtorno do Espectro do Autismo, acompanhadas em dois centros especializados na cidade de Maceió-AL, cuja amostra foi de 30 crianças com idades entre 2 e 11 anos, de ambos os gêneros, com diagnóstico de Transtorno do Espectro do Autismo leve. Ele teve início após a aprovação do Comitê de Ética e Pesquisa do Centro Universitário Cesmac, sob protocolo de $\mathrm{n}^{\circ}$ $647.531 / 2014$ e realizado após o responsável pela criança assinar o termo de consentimento livre e esclarecido.

A coleta dos dados ocorreu no período de agosto a setembro de 2014 , sendo realizada no Centro Unificado de Integração e Desenvolvimento do Autista, pertencente à Associação de Pais e Amigos dos ExcepcionaisMaceió/AL e no Núcleo de Assistência à Criança Especial. Esses locais foram escolhidos por serem considerados referência para o tratamento da criança com Transtorno do Espectro do Autismo de forma gratuita.

Inicialmente, as pesquisadoras entraram em contato com a responsável técnica da instituição para ter acesso ao cadastro de todas as crianças com Transtorno do Espectro do Autismo, para, posteriormente, verificar, tomando 
como base os critérios de inclusão, as crianças que iriam fazer parte da pesquisa. Dessa forma, foram selecionadas as crianças com diagnóstico clínico confirmado de Transtorno do Espectro do Autismo descrito em prontuário, de ambos os gêneros, com a faixa etária compreendida de 2 a 11 anos, que estivessem inseridas no programa de reabilitação e que fossem consideradas com diagnóstico leve, baseando-se na descrição do prontuário.

Foram excluídas as crianças com Transtorno do Espectro do Autismo que apresentavam alguma condição associada comprovada ao quadro de Transtorno do Espectro do Autismo, tais como Síndromes Genéticas ou doenças neurológicas.

Após os responsáveis pelas crianças serem esclarecidos sobre a importância do estudo e orientados sobre as etapas da pesquisa, foi aplicado um instrumento de coleta de dados composto de dados objetivos, visando obter informações sobre as características das mesmas como: gênero da criança, idade da criança, idade de diagnóstico, idade de início de tratamento, frequência à escola e terapias que realizam.

Posteriormente, foi realizada a avaliação das crianças com Transtorno do Espectro Autista por meio da escala de desenvolvimento motor de Rosa Neto $^{(9)}$, de acordo com a idade e de forma individual. A escala de desenvolvimento motor de Rosa Neto ${ }^{(9)}$ é indicada nos casos de crianças com atraso no desenvolvimento motor, com dificuldade na aprendizagem escolar, problemas de conduta e alterações neurológicas, mentais e/ou sensoriais.

As áreas de atuação dessa bateria de exame são: motricidade fina, motricidade global, equilíbrio, esquema corporal, organização espacial, organização temporal e lateralidade.

Considerou-se como variável primária, nesta pesquisa, o perfil psicomotor das crianças com Transtorno do Espectro Autista, que é representado pela idade motora geral e o quociente motor geral, ambos obtidos por meio da escala de desenvolvimento motor. Já as variáveis secundárias foram: idade, gênero, idade de diagnóstico e de início de tratamento, terapias que realizam, frequência à escola e escore da idade motora específica e do quociente motor geral específico.

A quantificação do escore das idades motoras é baseada na pontuação pré-determinada pela escala de desenvolvimento motor, segundo a qual, após a realização de cada prova motora, a criança recebe uma pontuação de 
acordo com o êxito da prova, onde a nota zero significa que não executou a

Ao término de todas as provas prova, meio $(1 / 2)$ que executou de maneira incompleta e um (1) que executou com êxito. Isso irá indicar a idade motora a que a criança pertence, em meses, e avalia a performance em relação à prova executada.

Depois da aplicação de cada prova específica, é realizado o somatório dos pontos para calcular a idade motora para cada elemento específico, padronizado em: idade motora para motricidade fina; idade motora para motricidade global; idade motora para equilíbrio; idade motora para esquema corporal; idade motora para organização espacial; idade motora para organização temporal. motoras, é realizado o cálculo do quociente motor específico e do Quociente motor geral que, por sua vez, é obtido por intermédio da divisão entre a idade motora geral e a idade cronológica em meses, multiplicado pela constante 100. Por meio desse resultado, a criança será classificada de acordo com a tabela pré-determinada por Rosa Neto ${ }^{(9)}$ em 7 níveis (muito superior, superior, normal alto, normal médio, normal baixo, inferior e muito inferior), baseando-se no resultado do Quociente motor geral (QMG), conforme quadro 01 abaixo.

Quadro 01 - Classificação dos Resultados da Escada de Desenvolvimento de Rosa Neto

\begin{tabular}{|c|c|c|}
\hline PONTUAÇÃO & CLASSIFICAÇÃO & FATOR DE RISCO \\
\hline 130 ou mais & MUITO SUPERIOR & NENHUM \\
\hline $120-129$ & SUPERIOR & NENHUM \\
\hline $110-119$ & NORMAL ALTO & NENHUM \\
\hline $90-109$ & NORMAL MÉDIO & NENHUM \\
\hline $80-89$ & NORMAL BAIXO & RISCO LEVE \\
\hline $70-79$ & INFERIOR & RISCO MODERADO \\
\hline 69 ou menos & MUITO INFERIOR & RISCO GRAVE \\
\hline
\end{tabular}

A aplicação da EDM foi realizada de uma única vez, em uma sala ampla, com clima adequado, sendo a aplicação do teste realizada em um período de 30 a 45 minutos, oferecendo as crianças um ambiente descontraído.
Destaca-se que o instrumento de coleta de dados e a EDM foram aplicados pelas pesquisadoras e, após a análise, os dados coletados foram armazenados em uma pasta eletrônica. $\mathrm{O}$ resultado de todas as baterias 
psicomotoras aplicadas foi entregue ao profissional responsável da instituição, com considerações e sugestões, bem como foi apresentado aos cuidadores o resultado de forma geral, obtido nesta pesquisa.

Os dados foram submetidos à análise descritiva, avaliando-se a distribuição de cada variável estudada por meio do programa utilizado, o Statistical Package for Social Sciences (SPSS) versão 17.0. Os resultados foram apresentados em gráficos e tabelas.

\section{Resultados}

Participaram desta pesquisa 30 crianças com TEA leve, sendo que $70 \%$ (21) eram do CUIDA e 30\% (9) do
NACE. Em relação aos gêneros das crianças, 93,3\% (28) eram do gênero masculino e $6,7 \%(2)$ do feminino. A idade do diagnóstico das crianças com TEA ocorreu com $4 \pm 1,8$ anos, variando de um ano até 8 anos.

No tocante à frequência à escola das crianças com TEA avaliadas, verificou-se que $93,3 \%$ frequentavam a escola e destas 100\% eram em escolas regulares.

A média de idade cronológica (IC) das crianças avaliadas foi de $88,5 \pm$ 27 em meses, o que corresponde aproximadamente a 7 anos aproximadamente.

$\mathrm{Na}$ tabela 01 estão descritas as características quanto ao programa reabilitacional das crianças com TEA avaliadas na pesquisa.

Tabela 01- Características Reabilitacionais das Crianças com Transtorno do Espectro Autista (TEA)

\begin{tabular}{lcc}
\hline \multicolumn{1}{c}{ VARIÁVEL } & N & \% \\
\hline Início das terapias & 1 & $3,3 \%$ \\
Até um ano antes do diagnóstico & 23 & $76,7 \%$ \\
No mesmo ano do diagnóstico & 6 & $20 \%$ \\
Mais de um ano do diagnóstico & 27 & $90 \%$ \\
Realiza Terapia Ocupacional & 27 & $90 \%$ \\
Realiza Fonoaudiologia & 19 & $63 \%$ \\
Realiza Fisioterapia & 27 & $90 \%$ \\
Realiza Psicologia & &
\end{tabular}

Fonte- Dados da Pesquisa. 
A média da IMG foi de $70 \pm 29,3$ em meses, sendo esta inferior à IC que foi de $88,5 \pm 27$ em meses. Os dados obtidos em relação às idades motoras de cada elemento psicomotor estão na tabela 02 , e o perfil psicomotor obtido, na figura 01 .

Tabela 02 - Idades Motoras Específicas (IMn) para cada Elemento Psicomotor e Idade Motora Geral (IMG) das Crianças com Transtorno do Espectro Autista (TEA) Avaliadas. IDADE EM MESES

\begin{tabular}{cccc}
\cline { 2 - 4 } PROVA MOTORA & Média & Mínimo & Máximo \\
\hline IM 1 & $70,2 \pm 36,1$ & 24 & 132 \\
IM 2 & $76,8 \pm 31,1$ & 24 & 132 \\
IM 3 & $77,6 \pm 35,89$ & 24 & 149,6 \\
IM 4 & $62 \pm 27,5$ & 0 & 132 \\
IM 5 & $76 \pm 31,6$ & 36 & 132 \\
IM 6 & $55,2 \pm 40,8$ & 0 & 132 \\
\hline IMG & $70 \pm 29,3$ & 24 & 132
\end{tabular}

Legenda - IM 1 (Motricidade fina), IM 2 (Motricidade Global); IM 3 (Equilíbrio); IM 4 (Esquema Corporal) ; IM 5 (Organização espacial ); IM 6 (Organização Temporal); IMG (Idade Motora Geral) Fonte- Dados da pesquisa.

Em relação à lateralidade das crianças com TEA avaliadas, verificou-se que $53,3 \%$ (16) possuíam lateralidade à direita, 33,33\% (10) cruzada e 13,3\%

(4) à esquerda.

Figura 01 - Perfil Psicomotor das Crianças com Transtorno do Espectro Autista (TEA).

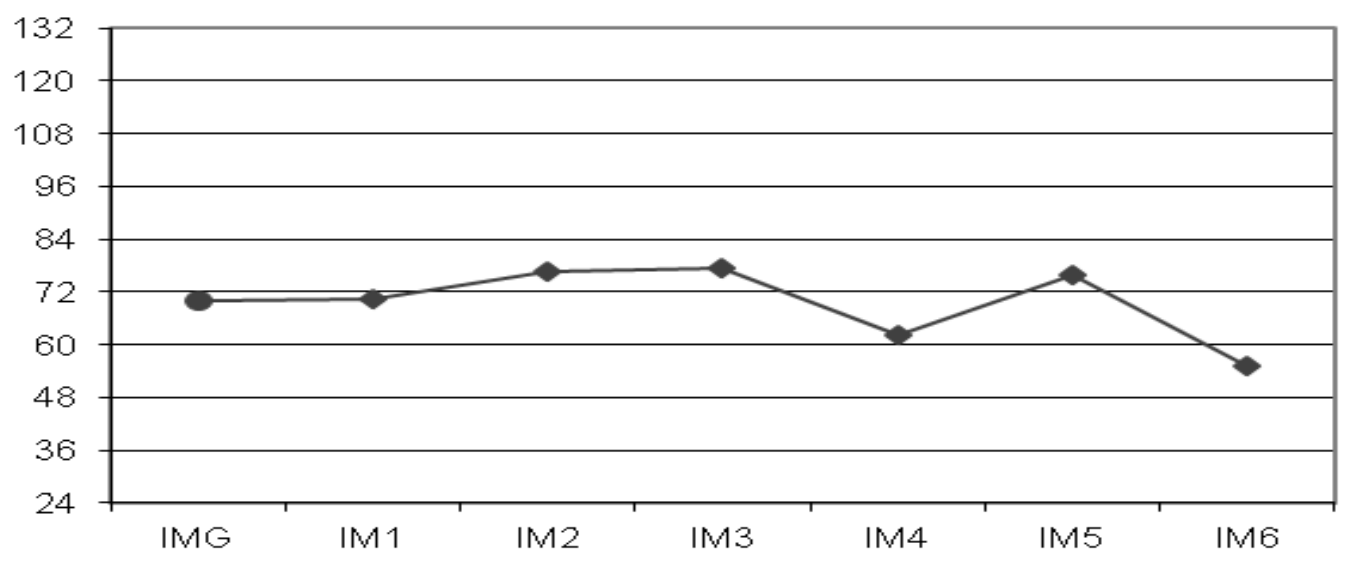

Legenda - IM 1 (Motricidade fina), IM 2 (Motricidade Global); IM 3 (Equilíbrio); IM 4 (Esquema Corporal); IM 5 (Organização espacial ); IM 6 (Organização Temporal); IMG (Idade Motora Geral)Fonte- Dados da pesquisa. 
Foi realizado o cálculo do QMn de cada prova motora, para se obter o QMG de cada criança avaliada. O escore médio do QMG foi de 77,3 $\pm 16,9$ sendo classificado em um nível inferior. Todavia estratificando o resultado, encontrou-se que $30 \%(9)$ eram classificadas em muito inferior, $26,7 \%$ (8) em inferior, $20 \%(6)$ em Normal Baixo , 3,3\% (1) em Normal Alto.

Portanto, as crianças com TEA avaliadas nesta pesquisa estavam compreendidas em muito inferior e inferior, utilizando a classificação prédeterminada por Rosa Neto.

\section{Discussão}

Os resultados do comprometimento motor nas habilidades motoras básicas em crianças com Transtorno do Espectro Autista têm sido observados já na primeira infância e nos primeiros 2 anos de $\operatorname{vida}^{(2)}$.

Comparando as amostras clínicas quanto às epidemiológicas encontra-se uma maior incidência de Transtorno do Espectro Autista em meninos do que em meninas, com proporções médias relatadas de cerca de 3,5 a 4 meninos para cada menina, acredita-se que, quando a criança com Transtorno do
Espectro Autista do gênero feminino for acometida, terá maior probabilidade de apresentar prejuízo cognitivo grave ${ }^{(10)}$.

Essas informações corroboram os dados coletados nesta pesquisa, visto que a incidência de meninos foi maior, havendo maior gravidade quando afetado o gênero feminino, sendo o critério de inclusão da pesquisa crianças de 2 a 11 anos com Transtorno do Espectro Autista leve. A escolha do critério de inclusão de acordo com a gravidade das crianças com Transtorno do Espectro Autista se deu pelo melhor desempenho cognitivo e aptidão para realização das etapas propostas na bateria psicomotora de Rosa Neto ${ }^{(9)}$.

A avaliação psicomotora tem por finalidade, fornecer aos educadores, profissionais clínicos e pesquisadores, informações pertinentes à prescrição de programas e atividades motoras, além da avaliação desses programas em crianças com desvios no sistema motor e problemas de desenvolvimento ${ }^{(11)}$.

Dessa maneira, como a maioria das crianças com Transtorno do Espectro Autista avaliadas nesta pesquisa frequentavam a escola, a avaliação psicomotora será considerada também um recurso que irá guiar o planejamento educacional das mesmas. Tendo em vista o objetivo primário que 
é o de melhorar as habilidades motoras, permitindo maiores níveis de funcionamento nas atividades da vida diária.

Nesta pesquisa, verificou-se que a maioria das crianças com Transtorno do Espectro Autista iniciaram o tratamento no mesmo ano do diagnóstico e foram diagnosticadas aproximadamente com 4 anos. Sabendo-se da importância do diagnóstico e intervenção precoce, fazse necessária a capacitação dos profissionais da saúde e médicos especialistas para o conhecimento $\mathrm{e}$ detecção dos primeiros sinais do Transtorno do Espectro Autista, promovendo, assim, uma melhor evolução das crianças.

O reconhecimento das primeiras manifestações das condições que afetam o desenvolvimento neuromotor permitirá que, quando necessário, as crianças com Transtorno do Espectro Autista sejam encaminhadas a diferentes especialistas para intervenções precoces que possam modificar o curso de seu prognóstico ${ }^{(12)}$.

Uma gama de aspectos pode retardar a intervenção, como é o caso da demora na detecção das primeiras dificuldades no comportamento da criança, na busca pela ajuda profissional e na realização do diagnóstico. De fato, alguns estudos têm demonstrado que crianças com Transtorno do Espectro Autista dificilmente recebem esse diagnóstico antes dos 5 anos, sendo que algumas são diagnosticadas apenas quando atingem idade escolar ${ }^{(12)}$. Tal fato pode ser verificado nesta pesquisa.

No trabalho da equipe multidisciplinar há a necessidade de uma inter-relação entre os diferentes profissionais que devem ver o paciente como um todo, numa atitude humanizada, trocando informações, de forma que o tratamento seja global, integrativo e interativo ${ }^{(13)}$.

Observou-se no presente estudo a presença da equipe multidisciplinar em ambas instituições, composto por Terapeuta Ocupacional, Fonoaudiólogo, Psicólogo, Fisioterapeuta, Psicopedagogo e Médicos especialistas.

Vários estudos não conseguiram encontrar diferenças motoras entre crianças com Transtorno do Espectro Autista e aquelas com dificuldades de aprendizagem ou deficiência intelectual , atraso no desenvolvimento geral, e distúrbios de linguagem. Isso pode ser em decorrência da variabilidade de quadros que as crianças com Transtorno do Espectro Autista podem apresenta ${ }^{(2)}$. Todavia, nesta pesquisa identificou um perfil psicomotor alterado nas crianças com Transtorno do Espectro Autista. Isso porque a Idade Motora Geral das 
crianças com Transtorno do Espectro

Autista avaliadas neste estudo foi na sua média, inferior à sua idade cronológica , indicando, dessa forma, um comprometimento das funções motoras, bem como uma desarmonia entre os elementos psicomotores.

Por meio da análise da tabela 02 e da figura 01, verificou-se que as crianças com Transtorno do Espectro Autista avaliadas obtiveram menor idade motora no Esquema Corporal que corresponde a idade motora especifica 4 e Organização Temporal que corresponde a idade motora especifica 6 ; e a maior idade motora na Motricidade Global que corresponde a idade motora 2 , equilíbrio, que corresponde a idade motora 3 e Organização Espacial que corresponde a idade motora 5.

Machado $^{(14)}$ relata, que, uma das formas de se trabalhar o desenvolvimento global do indivíduo e os possíveis desvios é por meio da intervenção psicomotora, área de atuação do profissional de Fisioterapia. Assim, a Fisioterapia pode atuar na criança com Transtorno do Espectro Autista na ativação dos níveis sensorial e motor, buscando melhorar a concentração, a memória e as habilidades motoras, como a coordenação e o equilíbrio.
Apesar da existência da equipe multidisciplinar no tratamento das crianças com Transtorno do Espectro Autista, foi detectado que apenas 19 $(63 \%)$ dessas crianças realizam Fisioterapia.

Segundo Mendes ${ }^{(15)}$ para se conhecer a Psicomotricidade, deve-se saber sobre as condutas funcionais que se referem àquelas cuja ação, qualidade e mensuração são possíveis de serem percebidas e que conjuntamente formam a integralização motora.

Para Fonseca ${ }^{(16)}$, a lateralidade constitui um processo essencial às relações entre a motricidade e a organização psíquica intersensorial. Representando a conscientização integrada e simbolicamente interiorizada dos dois lados do corpo, lado esquerdo e lado direito, o que pressupõe a noção da linha média do corpo.

No estudo de Bosquerolli ${ }^{(17)}$, realizado com uma criança com Transtorno do Espectro Autista no estado de Santa Catarina, utilizando a Escala de Desenvolvimento Motor de Rosa Neto, o resultado encontrado na avaliação qualitativa inicial foi um nível "muito inferior" de acordo com o Manual de Avaliação Psicomotora. Todavia, após a intervenção por três meses a mesma não mudou de 
classificação. Porém, apresentou uma melhora significativa nos quociente motora geral, Equilíbrio e Esquema Corporal.

Nesta pesquisa, as crianças com Transtorno do Espectro Autista avaliadas estavam compreendidas na sua maioria na classificação de muito inferior e inferior, dado semelhante ao encontrado no estudo de Bosquerolli ${ }^{(17)}$.

Já no estudo de Schilling e Rosa Neto ${ }^{(18)}$, realizado com uma criança com Transtorno do Déficit de Atenção e Hiperatividade, utilizando, também, a Escala de Desenvolvimento Motor de Rosa Neto, ocorrido no Centro de Educação Física, Fisioterapia e Desportos de Santa Catarina, verificouse que as crianças apresentaram inicialmente um resultado qualitativo "inferior" no quociente motor geral. Todavia, após a intervenção realizada, em 25 sessões, a criança passou para o nível de "Normal baixo". Os dados citados não corroboram este estudo, onde a maioria das crianças foi classificada como muito inferior $\mathrm{e}$ inferior, classificando-se apenas 20\% como normal baixo e 3,3\% normal alto.

No estudo de Rosa Neto et al, ${ }^{(19)}$ realizado com crianças com dificuldade de aprendizagem, no Núcleo Interdisciplinar de Apoio ao Desenvolvimento Humano em
Florianópolis, utilizando a escala de desenvolvimento motor, em relação aos quociente motor avaliados, todos apresentaram déficit em relação à idade cronológica sendo que a Equilíbrio e Organização temporal as que apresentaram maiores alterações, sendo as crianças classificadas como "muito inferiores". Nesta pesquisa, dado semelhante foi encontrado em relação ao elemento psicomotor Organização Temporal.

É importante mencionar que esta pesquisa não realizou uma intervenção psicomotora nas crianças com Transtorno do Espectro Autista, apenas buscou conhecer o perfil psicomotor das mesmas. Após esse perfil ter sido traçado, as pesquisadoras propuseram um protocolo de intervenção psicomotora a ser realizado nas crianças com Transtorno do Espectro Autista avaliadas nessa pesquisa como desmembramento dessa pesquisa.

\section{Conclusão}

O perfil psicomotor médio encontrado nas crianças com Transtorno do Espectro Autista avaliadas nesta pesquisa demonstrou que as mesmas estão em um nível inferior em relação ao desenvolvimento dos aspectos psicomotores esperados para a sua idade 
cronológica. Verificou-se também a presença de déficits em todos os elementos psicomotores, sendo os mais comprometidos o esquema corporal e a organização temporal. E os menos comprometidos Motricidade Global, Equilíbrio e Organização Espacial.

Dessa forma, o Fisioterapeuta pode lançar mão da Psicomotricidade e estimular não só o elemento motor falho, mas também todos eles, promovendo uma integração de seus componentes através de uma terapia psicomotora adequada, visando, inclusive, uma reeducação na organização das sensações, percepções e otimização da cognição.

\section{Referências}

1. Backes B, Zanon RB, Bosa CA. A relação entre regressão da linguagem e desenvolvimento sociocomunicativo de crianças com transtorno do espectro do autismo. CoDAS. (São Paulo) [periódico na Internet]. 2013 [citado 2014 set. 20]; 25(3): 268-73. Disponível em: http:/www.scielo.br/ scielo.php?script $=$ sci_arttext\&pid $=$ S23 17-17822013000300013.

2. Radonovich KJ, Fournier KA, Hass CJ. Relationship between postural control and restricted, repetitive behaviors in autism spectrum disorders. Front Integr Neurosci. [internet newspaper]. 2013 [quoted 2014 sep. 26]; 7:7-28. Disponível em: https://www.ncbi.nlm.nih.gov/pubmed/ 23675326.

3. Simões JR, Murijo MG, Pereira K. Perfil psicomotor na praxia global e fina de crianças de três a cinco anos pertencentes à escola privada e pública. ConScientiae Saúde (São Paulo) [periódico na Internet]. 2008 [citado 2014 set. 29]; 7(2): 151-57. Disponível em: http://www.redalyc. org/articulo.oa? id=92970203.

4. Burns YR, MacDonald J. Desenvolvimento da motricidade desde o nascimento até os 2 anos de idade. In: Fisioterapia e crescimento na infância. Fisioterapia (São Paulo) [periódico na Internet]. 1999 [citado 2014 set. 20]; 31-42. Disponível em: http://www2.pucpr.br/reol/index.p hp/RFM?dd1=2820\&dd99=pdf.

5. Klin AMI, Chawarska ER, Volkmar F. Avaliação clínica de crianças com risco de autismo. Educação (Porto Alegre) [periódico na Internet]. 2006 [citado 2014 set. 25]; 1 (58): 255-97. Disponível em: http://revistaseletroni cas.pucrs.br/ojs/index.php/faced/article /download/433/329.

6. Mello AM. Psicomotricidade Elementos Fundamentais. In. 6. Mello AM. Psicomotricidade, Educação Física e Jogos Infantis. São Paulo: Ibrasa; 2002; 4: 21-30.

7. Figueira MMA. Assistência fisioterápica à criança portadora de cegueira congênita. Fisioterapia [periódico na Internet]. 2000 [citado 2014 set. 25]; 17: 4-30. Disponível em: http://www.ibc.gov.br/images/conteud o/revistas/benjamin constant/2000/edi cao-17-dezembro/Nossos Meios RBC _RevDez2000_ARTIGO2.pdf. 
8. Brumer A, Pavei K, Mocelin DG, Pasinato LA, Santos PJM, Santos MAM. Saindo da "escuridão": perspectivas da inclusão social, econômica, cultural e política dos portadores de deficiência visual em Porto Alegre. Sociologias (Porto Alegre) [periódico na Internet]. 2004 [citado 2014 set. 29]; 6(11): 300-27. Disponível em: http://www.seer.ufrgs .br/sociologias/article/download/5453/ 3102 .

9. Rosa NF. Manual de avaliação motora. Florianópolis: DIOESC; 2014; 2 .

10. Ami K. Autismo e síndrome de Asperger: uma visão geral. Rev. Bras. Psiquiatr. 2006;28 (Supl I); S3-S11.

11. Rosa GKB, Marques I, Papst JM, Gobbi LTB. Desenvolvimento motor de criança com paralisia cerebral: avaliação e intervenção. [periódico na Internet]. 2008 [citado 2014 out. 02]; 14(2):163-76. Disponível em: http://www.scielo.br/scielo.php?script $=$ sci_arttext\&pid $=$ S1413-65382008000 200002

12. Zanon RB, Backes B, Bosa CA. Identificação dos primeiros sintomas do autismo pelos pais. Psicologia (Brasília) [periódico na Internet]. 2014 [citado 2014 out. 05]; 30 (1):25-3. Disponível em: http://www.scielo.br/ scielo.php?script $=$ sci_arttext\&pid $=\mathrm{S} 01$ 02-37722014000100004.

13. Núcleo Reviver. (Goiânia) [periódico na Internet]. 2012 [citado 2014 out. 07]. Disponível em: http://www.nucleoreviver.com/.
14. Andrade MP. Autismo e integração sensorial - A intervenção psicomotora como um instrumento facilitador no atendimento de crianças adolescentes autistas. Minas Gerais. [Dissertação de mestrado]. Universidade Federal de Viçosa - MG; 2013.

15. Cacemiro KP, Pires ER, Psicomotricidade e sua relação com a Fisioterapia. [trabalho apresentado no III Simpósio Internacional de Ciências Integradas da Unaerp Campus Guarujá; 2006; São Paulo; Brasil]. Disponível em: http://www.unaerp.br /index.php/documentos/982-psicomotri cidade-e-sua-relacao-com-a-fisioterapi a/file.

16. Pacher LAG. Lateralidade e Educação física. Santa Catarina. Monografia; Instituto Catarinense de Pós-Graduação - Curso de Educação Física; 2013. Disponível em: http://webcache.googleusercontent.co $\mathrm{m} /$ search? $\mathrm{q}=$ cache:NZC3hMqlmL4J:ce v.org.br/biblioteca/lateralidade-passefutebol-academicos-curso-educacao-fis ica-uma-universidade-publica-santa-ca tarina $/+\& \mathrm{~cd}=1 \& \mathrm{hl}=\mathrm{pt}-\mathrm{BR} \& \mathrm{ct}=\mathrm{clnk} \& \mathrm{~g}$ $\mathrm{l}=$ br.

17. Prestes DB, Amaro KN, Arab C, Neto FR. Avaliação e intervenção motora com uma criança autista. (Santa Catarina) [periódico na Internet]. 2009 [citado 2014 out. 10]; 14 (138):1. Disponível em: http://www.efdeportes.com/efd138/inte rvencao-motora-com-uma-crianca-auti sta.htm.

18. Poeta LS, Neto FR. Intervenção motora em uma criança com transtorno do déficit de atenção/hiperatividade (TDAH). (Santa Catarina) [periódico na Internet]. 2005 [citado 2014 out. 10]; 10 (89):1. Disponível em: http://www.efdeportes.com/efd89/tdah. htm. 
19. Neto FR, Costa SH, Poeta LC.

Perfil motor em escolares com problemas de aprendizagem. Pediatria

Moderna (Santa Catarina) [periódico

na Internet]. 2005 [citado 2014 out.

12]; 61 (2) 2: 109-17. Disponível em:

http://www.moreirajr.com.br/revistas.a

sp? fase $=$ r003\&id_materia $=2975$. 